\title{
Aplicaciones de la Teoría de Colas a la provisión óptima de servicios sociales: El caso del servicio de Teleasistencia
}

\section{FRANCISCO J. PELÁEZ FERMOSO}

Departamento de Economía Aplicada, UNIVERSIDAD DE VALLADOLID, ESPAÑA. Email:ppelaez@eco.uva.es

\section{JESÚS MARÍA GÓMEZ GARCÍA}

Departamento de Economía Aplicada, UNIVERSIDAD DE VALLADOLID, ESPAÑA. Email: jmgomez@eco.uva.es

\author{
ANA GARCÍA GONZÁLEZ \\ Departamento de Economía Aplicada, UNIVERSIDAD DE VALLADOLID, ESPAÑA. E- \\ mail: anagar@eco.uva.es
}

\begin{abstract}
RESUMEN
En este trabajo se analiza la eficiencia en la provisión del servicio de Teleasistencia para las personas dependientes de 65 y más años. Para ello, se han estimado los costes que optimizan su funcionamiento según un modelo de Teoría de Colas. La aplicación de esta metodología se lleva a cabo tomando como caso de estudio el servicio de Teleasistencia en Castilla y León, por ser ésta una de las regiones españolas con mayor tasa de envejecimiento demográfico. Para evaluar el impacto del envejecimiento demográfico sobre la demanda del servicio de Teleasistencia, se utilizan las proyecciones de población del INE ajustadas a la población anciana dependiente para el período 2005-2015.
\end{abstract}

Palabras clave: Población anciana dependiente, servicio de Teleasistencia, eficiencia de costes, teoría de colas.

\section{Queuing Theory Applications to an Optimal Provision of Social Services: The Telecare Service Case}

\begin{abstract}
In this paper we analyse the efficiency of Telecare service management for dependent people over 65 years old. Accordingly, we estimate the costs which optimize the operation of the Telecare service, based on a Queuing Theory model. The methodological application is carried out using the Telecare service in Castilla y León as study case, given that it is one of the Spanish regions with higher rate of demographic aging. In order to measure the impact of demographic ageing on the Telecare service demand, we consider the INE population projections adjusted for dependent elderly population to the time period 2005-2015.
\end{abstract}

Keywords: Dependent Elderly Population, Telecare Service, Cost Efficiency, Queuing Theory.

Clasificación JEL: C44, I38, O18

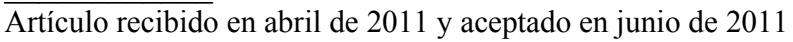

Artículo disponible en versión electrónica en la página www.revista-eea.net, ref. ə-29301 


\section{INTRODUCCIÓN}

Un hecho constatado en la sociedad española y en la mayoría de los países de la Unión Europea es el progresivo envejecimiento de su población y el consiguiente incremento de gastos derivados de su atención (OCDE, 2001; Moragas et al., 2003, Gómez et al., 2005). Este cambio demográfico resulta especialmente relevante por cuanto que al aumentar los estratos de población con edades superiores a los 65 años, en los que es mucho mayor la probabilidad de caer en estado de dependencia ${ }^{1}$ debido a una posible pérdida de capacidad física o psíquica, va a traer como consecuencia que un mayor número de personas ancianas tenga que recurrir a la ayuda de otras para poder llevar a cabo los principales actos de la vida cotidiana (Puga y Abellán, 2001; Pérez, 2006). Esta circunstancia, unida a otros factores sociales como el cambio en las estructuras familiares, se considera un factor causal del aumento en la demanda de servicios de atención y ayuda a las personas dependientes en los últimos años (Informe Pfizer, 2002; Jiménez, 2004). En este contexto, los recursos que tienen que asignarse a las necesidades de la población dependiente serán cada vez más cuantiosos y, también más compleja su organización.

Para hacer frente a este problema, el Sistema para la Autonomía y Atención a las Personas en Situación de Dependencia (SAAD), que comienza en España a partir del año 2007 y que terminará de implantarse en el año 2015, establece un proceso gradual de protección por medio de distintos tipos de servicios sociales puestos a disposición de la población dependiente. Ello hace de la atención a la dependencia un nuevo campo de la intervención pública que va a poner a prueba la capacidad de la sociedad y de sus instituciones para adaptarse y dar soluciones a esta nueva realidad (Casado y López, 2004; Monteverde, 2004; López et al., 2005).

En el Libro Blanco de la Dependencia (IMSERSO, 2005) y otros estudios (Rodríguez y Ferreiro, 1997; Cruz Roja Española, 2002; Sancho y Díaz, 2007) se analizan los efectos económicos derivados de la puesta en funcionamiento del SAAD considerando distintos tipos de recursos sociales (residencias, centros de día, ayuda informal, servicio de ayuda a domicilio y el servicio de Teleasistencia) para la población dependiente, según grados de dependencia ${ }^{2} ; \mathrm{y}$

\footnotetext{
${ }^{1}$ Una persona es considerada dependiente si por causa de la edad, la enfermedad, la discapacidad o cualquier otra problemática física, psíquica o sensorial, necesita ayuda para poder realizar algunas o todas las actividades básicas de la vida diaria (ABVD).

${ }^{2}$ La situación de dependencia se clasifica en los siguientes grados: Grado I (Dependencia Moderada), cuando la persona necesita ayuda para realizar varias de las actividades básicas de la vida diaria, al menos una vez al día; Grado II (Dependencia Severa), si necesita ayuda para el mismo fin dos o tres veces al día; y Grado III (Gran Dependencia), si precisa ayuda para la misma finalidad varias veces al día y la colaboración de una persona de forma permanente. A su vez, cada uno de estos grados de dependencia se clasifican en dos niveles, Nivel I y Nivel II, según la autonomía de la persona y la intensidad del cuidado que requiera.
} 
más concretamente, uno de los servicios de atención que se espera tenga más demanda en el futuro es el de Teleasistencia domiciliaria (Sánchez-Criado y López, 2007).

El servicio de Teleasistencia tiene por objetivo mantener a las personas en situación de dependencia en contacto con su entorno socio-familiar y sin necesidad de que tenga que ingresar en un centro residencial para cubrir sus necesidades de atención (Cruz Roja Española, 2002; López, 2008; Tirado et al., 2008). En concreto, este servicio permite al usuario ${ }^{3}$ estar en contacto a través de la línea telefónica con un Centro de Atención especializado, pulsando el botón de un medallón o de un colgante remoto que llevan consigo constantemente y desde el que se podrá generar una alarma desde cualquier parte del domicilio, o bien a través de un teléfono especial pulsando una tecla y sin necesidad de descolgar el auricular. También puede disponer de unidades móviles que, en caso de necesidad, se trasladan al domicilio del usuario para dar solución a la emergencia surgida ${ }^{4}$. En España, el servicio de Teleasistencia está gestionado por Cruz Roja Española ${ }^{5}$.

Este trabajo se ha estructurado en varios apartados. Después de esta introducción, en el segundo epígrafe se describen los objetivos y la metodología aplicada en el análisis que se llevará a cabo con posterioridad. A continuación, en el epígrafe tercero, se realiza una estimación de la población anciana dependiente en Castilla y León para el período considerado, que va a representar la demanda potencial del servicio de Teleasistencia domiciliaria en dicha región. En el epígrafe cuarto se plantea el modelo de colas que será utilizado para estimar los costes de la prestación de este servicio y se resumen los resultados que se obtienen de su simulación. El quinto epígrafe comprende un análisis de

\footnotetext{
${ }^{3}$ Los destinatarios de este servicio deberán reunir deterrminados requisitos, tales como ser personas dependientes que se encuentren en situación de riesgo psico-social o físico al vivir gran parte del día solas; no padecer trastornos mentales graves, incluidas las demencias seniles; no padecer deficiencias importantes de audición y/o expresión oral y disponer en su domicilio de línea telefónica y de suministro eléctrico.

${ }^{4}$ El Programa de Teleasistencia Domiciliaria se ha instrumentado en España en 1993 a través de un Convenio de Cooperación suscrito entre el IMSERSO y la Federación Española de Municipios y Provincias (FEMP). A este programa se van adhiriendo, a través de convenios de colaboración, las Corporaciones Locales interesadas en su implantación. La evolución del Servicio de Teleasistencia Domiciliaria ha sido muy relevante en la última década, pasando de 7070 usuarios en el año 1995 a 261433 a comienzos de 2006. De esta manera se ha alcanzado un índice de cobertura del 3,5\% sobre las personas mayores de 65 años y aproximadamente el $11 \%$ sobre el total de personas mayores que viven solas. Por Comunidades Autónomas destaca el índice de cobertura en Madrid (9,2\%) y en el extremo opuesto Canarias $(0,97 \%)$, ocupando Castilla y León una posición intermedia (3,01\%).

${ }^{5}$ En la Comunidad Autónoma de Castilla y León, la prestación de este Servicio de Teleasistencia se financia conjuntamente por la Junta de Castilla y León, la Federación Española de Municipios y Provincias (FEMP), los Ayuntamientos y las Diputaciones Provinciales de la región.
} 
sensibilidad del modelo respecto de las variables más relevantes. Finalmente, se recogen las principales conclusiones extraídas de los resultados de la simulación del modelo de colas aplicado, así como las referencias bibliográficas utilizadas en este trabajo.

\section{OBJETIVOS Y METODOLOGÍA}

Con la realización de este trabajo se pretende analizar la eficiencia del servicio de Teleasistencia que atiende a las personas ancianas dependientes desde la perspectiva de los costes económicos que optimizan su funcionamiento, tomando como referencia el caso de la Comunidad Autónoma de Castilla y León. Para ello, se planteará un modelo matemático fundamentado en la Teoría de Colas. Esta metodología resulta especialmente adecuada para analizar las situaciones en las que existen un número importante de usuarios que demandan la prestación de un determinado servicio y pueden producirse situaciones de espera o colas, pues el dilema entre la eficacia (dar un buen servicio) y la eficiencia (hacerlo con pocos recursos) es universal (Singer et al., 2008). En este sentido, los modelos de colas permiten a los gestores del mismo la toma racional de decisiones considerando en el modelo tanto la capacidad del servicio como los indicadores de gestión o desempeño de interés para el administrador (número medio de usuarios, probabilidad de estar ocupado un operador, etc.) y otros de especial relevancia para el usuario (tiempos de espera en ser atendido, etc.). Para la aplicación de este modelo se precisa, no obstante, conocer o estimar la distribución de probabilidad que siguen los tiempos medios entre las llamadas de los usuarios y la distribución que siguen los tiempos medios que tardan estas personas en ser atendidas.

A través de la simulación del modelo de colas planteado, se estima el número de operadores de comunicaciones que minimizan los costes totales por usuario y que optimizan el funcionamiento del servicio de Teleasistencia en Castilla y León, teniendo en cuenta la demanda que realizan las personas dependientes de 65 y más años durante el horizonte temporal 2005-2015. Los resultados del estudio podrán, asimismo, ser contrastados con los datos reales suministrados por Cruz Roja Española, institución que gestiona este servicio, para determinar las posibles desviaciones en términos de eficiencia.

Las fuentes estadísticas utilizadas para la realización de este trabajo han sido las siguientes: las proyecciones de la población de España para el Escenario-1 del INE (2005), obtenidas a partir del Censo de Población de 2001 para el período 2005-2015; la Encuesta sobre Discapacidades, Deficiencias y Estados de Salud, EDDES (INE, 2002), de cuya explotación se han obtenido las tasas de población dependiente para dicha Comunidad Autónoma y que, combinadas con las anteriores proyecciones, permiten deducir las proyecciones de la población anciana dependiente regional para dicho período; las tablas salariales revisadas 
según Convenio Colectivo para Castilla y León; y los datos proporcionados por Cruz Roja Española que gestiona dicho servicio de Teleasistencia en la Comunidad Autónoma de Castilla y León.

De acuerdo con los objetivos descritos previamente, el proceso metodológico seguido en este trabajo comprendería:

- La proyección de la población anciana dependiente en la Comunidad Autónoma de Castilla y León para el período 2005-2015, teniendo en cuenta distintos grados de dependencia.

- El planteamiento de un modelo matemático según la Teoría de Colas que será utilizado para representar el funcionamiento del Servicio de Teleasistencia en Castilla y León; y de cuya simulación se obtendrán los valores que alcanzan las distintas variables que caracterizan la prestación de este servicio en la región.

- La estimación, a través del modelo de colas, del número de operadores de comunicaciones que minimizan los costes totales por usuario vinculados a la atención de las personas dependientes demandantes de este servicio.

- Un análisis de sensibilidad del coste total por usuario ante pequeñas variaciones en los valores de algunos parámetros del modelo de colas.

\section{ESTIMACIÓN DE LA POBLACIÓN POTENCIALMENTE DEMANDANTE DEL SERVICIO DE TELEASISTENCIA}

En este epígrafe se realiza un estudio prospectivo de la población anciana dependiente en Castilla y León para el período 2005-2015, que constituye la demanda potencias del servicio de Teleasistencia. Para ello, en primer, se precisa estimar la población anciana dependiente. Las proyecciones demográficas para la población de 65 y más años en Castilla y León, por grupos de edad y sexo, han sido obtenidas a partir de las proyecciones para la población española publicadas por el INE para el período 2002-2017. Las proyecciones utilizadas contemplan las hipótesis ${ }^{6}$ que configuran el denominado Escenario 1 (INE, 2005).

Por otra parte, de la explotación de los datos que recoge la Encuesta sobre Discapacidades, Deficiencias y Estados de Salud (EDDES) que elabora el INE,

\footnotetext{
${ }^{6}$ Concretamente, las hipótesis que, sobre la evolución futura de los tres fenómenos demográficos (natalidad, mortalidad y migraciones), se consideran en el Escenario 1 se apoyan, fundamentalmente, en las cifras observadas sobre cada uno de ellos en el pasado. No obstante, en cuanto las migraciones, dicho Escenario 1 contempla que las entradas netas de extranjeros en España evolucionan según la tendencia más reciente hasta el año 2010, año a partir del cual se mantienen constantes.
} 
se han deducido las tasas de dependencia ${ }^{7}$ para las personas ancianas de la región, diferenciando por grupos de edad y sexo, así como considerando diferentes grados de dependencia. Para ello, previamente, se hace necesario estimar las tasas de discapacidad a partir de la información que recoge la $\mathrm{EDDES}^{8}$ sobre el número de horas de atención que necesitan las personas discapacitadas.

\subsection{Estimación de las tasas de discapacidad y de dependencia}

La Encuesta sobre Discapacidades, Deficiencias y Estados de Salud (INE, 2002) permite determinar la población anciana con al menos una discapacidad y que manifiesta, además, recibir cierto número de horas de cuidados a la semana, según el grupo de edad y sexo. Dichas tasas de discapacidad se recogen en el Cuadro 1.

\section{Cuadro 1}

Tasas de Discapacidad para personas de 65 y más años Comunidad Autónoma de Castilla y León

\begin{tabular}{|l|c|c|c|c|c|c|}
\hline Grupos de Edad/Sexo & Total & $\mathbf{6 5}$ a 69 & $\mathbf{7 0}$ a $\mathbf{7 4}$ & $\mathbf{7 5}$ a 79 & $\mathbf{8 0}$ a $\mathbf{8 4}$ & $\mathbf{8 5}+$ \\
\hline Ambos Sexos & 0,37270 & 0,21083 & 0,28184 & 0,43905 & 0,51021 & 0,71406 \\
\hline Población Masculina & 0,32349 & 0,20671 & 0,24330 & 0,38731 & 0,49549 & 0,60679 \\
\hline Población Femenina & 0,41058 & 0,21452 & 0,31428 & 0,47887 & 0,51927 & 0,77192 \\
\hline
\end{tabular}

Fuente: Elaboración propia a partir de los datos de la EDDES.

Una vez determinada la población discapacitada en la Comunidad Autónoma de Castilla y León, y considerando el número de personas que declaran recibir horas semanales de atención en la $\operatorname{EDDES}^{9}$, se obtienen las tasas de dependen-

${ }^{7}$ Las tasas de dependencia se definen como el cociente entre el número de personas ancianas discapacitadas que reciben atención y el total de personas ancianas discapacitadas para cada grupo de edad y sexo.

${ }^{8}$ Este criterio supone aceptar implícitamente que existe homogeneidad entre quienes prestan la ayuda a la persona dependiente así como en la naturaleza de los cuidados recibidos por ésta, por no estar disponible esta información en la EDDES. El supuesto de asociar el grado de dependencia a un número de horas de atención recibida es común entre los estudios de este tipo. Otro posible baremo o escala para la medición del grado de dependencia es tomar como criterio de referencia las actividades básicas de la vida diaria (ABVD), si bien no existe una clasificación única de dichas actividades.

${ }^{9}$ La variable de la EDDES que proporciona información sobre el número de horas de atención a la semana es HORASSEM. El grado de dependencia asociado al número de horas semanales de atención que demandan dichas personas es el siguiente: De 7 a 14 horas, dependencia moderada; de 15 a 40 horas, dependencia severa, y más de 40 horas, gran dependencia. 
cia $^{10}$ de la población anciana de la región por grupos de edad y sexo, que se recogen en el Cuadro 2.

Dichas tasas específicas de dependencia, aplicadas a los resultados de la proyección de la población discapacitada de la región, permiten obtener la población dependiente por grupos de edad, sexo y también por grados de dependencia de la Comunidad Autónoma de Castilla y León para el período 20052015, que es horizonte temporal objeto de análisis.

\section{Cuadro 2}

Tasas de Dependencia para personas de 65 y más años

Comunidad Autónoma de Castilla y León

\begin{tabular}{|l|c|c|c|c|c|c|}
\hline Grupos de Edad / Sexo & Total & $\mathbf{6 5}$ a $\mathbf{6 9}$ & $\mathbf{7 0}$ a $\mathbf{7 4}$ & $\mathbf{7 5}$ a $\mathbf{7 9}$ & $\mathbf{8 0}$ a $\mathbf{8 4}$ & $\mathbf{8 5}+$ \\
\hline Población Masculina & $\mathbf{0 , 3 1 6 0}$ & 0,2414 & 0,1853 & 0,2721 & 0,3838 & 0,5564 \\
\hline Población Femenina & $\mathbf{0 , 5 0 1 6}$ & 0,3415 & 0,4424 & 0,4211 & 0,5191 & 0,7056 \\
\hline
\end{tabular}

Fuente: Elaboración propia a partir de los datos de la EDDES.

De acuerdo con el Libro Blanco de la Dependencia (IMSERSO, 2005), la demanda de diferentes servicios de atención a las personas ancianas dependientes (residencias, centros de día, ayuda a domicilio, Servicio de Teleasistencia, atención informal) estará en función del grado de dependencia que presenten. Igualmente, se formulan una serie de hipótesis a fin de determinar el número de personas dependientes que pueden solicitar esos servicios en el período temporal considerado.

\subsection{Hipótesis de demanda de servicios}

Los supuestos sobre la utilización de los diferentes recursos asistenciales por la población dependiente han sido extraídos del Libro Blanco de la Dependencia ${ }^{11}$ (IMSERSO, 2005) y se recogen en la Cuadro 3. Se comprueba que, en general, los porcentajes de demanda de servicios decrecen con la edad y con el grado de severidad de las personas dependientes.

10 Siguiendo la metodología de Monteverde (2004), las tasas de dependencia se suponen constantes para todo el horizonte temporal analizado.

${ }^{11}$ Si bien en el Libro Blanco de la Dependencia se describen las hipótesis de demanda de servicios para la población anciana dependiente hasta el año 2010, en este estudio hemos considerado que dichas hipótesis se mantienen constantes por cuanto no cabe esperar cambios sustanciales en los porcentajes de demanda de servicios para tan corto período temporal y en el que aún tiene que terminar de implantarse el SAAD. 


\section{Cuadro 3}

Hipótesis de Demanda de Servicios para la Población Dependiente de 65 y más años según Grados de Dependencia y Grupos de Edad (\%)

\begin{tabular}{|l|c|c|}
\hline \multirow{2}{*}{$\begin{array}{c}\text { Años/Grupos de Edad } \\
\text { Grados Dependencia }\end{array}$} & \multicolumn{2}{|c|}{ Años 2005-2015 } \\
\cline { 2 - 3 } & $\mathbf{6 5}$ a 79 años & $\mathbf{+ 8 0}$ años \\
\hline Dependencia Moderada & 100.0 & 100.0 \\
\hline Dependencia Severa & 75.0 & 50.0 \\
\hline Gran Dependencia & 15.0 & 6.0 \\
\hline
\end{tabular}

Fuente: Elaboración propia a partir de los datos del Libro Blanco de la Dependencia (2004).

De este modo, aplicando estos porcentajes, según la edad y el grado de dependencia, a los datos de la población anciana dependiente (Cuadro 4), se obtiene la población dependiente de 65 y más años que constituye el colectivo potencialmente demandante del servicio de Teleasistencia en ámbito territorial objeto de estudio (Cuadro 5).

\section{Cuadro 4}

Población Dependiente de 65 y más años (ambos sexos).

Comunidad Autónoma de Castilla y León (2005-2015). Escenario 1

\begin{tabular}{|c|c|c|c|c|c|c|c|c|c|c|c|c|}
\hline \multicolumn{13}{|c|}{ Tasas de Discapacidad y Tasas de Dependencia constantes } \\
\hline $\begin{array}{l}\text { Grupos } \\
\text { de Edad }\end{array}$ & $\begin{array}{c}\text { Grados de } \\
\text { Dependencia }\end{array}$ & 2005 & 2006 & 2007 & 2008 & 2009 & 2010 & 2011 & 2012 & 2013 & 2014 & 2015 \\
\hline \multirow{4}{*}{65 a 79} & Moderada & 20552 & 20326 & 20098 & 19888 & 19638 & 19386 & 19094 & 18803 & 18495 & 18219 & 17934 \\
\hline & Severa & 7116 & 7064 & 6999 & 6931 & 6841 & 6749 & 6633 & 6508 & 6368 & 6240 & 6095 \\
\hline & G. Dependencia & 13129 & 13033 & 12911 & 12773 & 12602 & 12411 & 12190 & 11975 & 11756 & 11562 & 11366 \\
\hline & Total & 40797 & 40423 & 40009 & 39592 & 39081 & 38547 & 37917 & 37287 & 36619 & 36020 & 35395 \\
\hline \multirow{4}{*}{$80+$} & Moderada & 19445 & 20241 & 21082 & 21933 & 22804 & 23657 & 24499 & 25300 & 26064 & 26767 & 27433 \\
\hline & Severa & 12762 & 13315 & 13925 & 14566 & 15223 & 15875 & 16513 & 17127 & 17713 & 18268 & 18794 \\
\hline & G. Dependencia & 24367 & 25417 & 26568 & 27769 & 29001 & 30222 & 31421 & 32572 & 33670 & 34708 & 35688 \\
\hline & Total & 56575 & 58973 & 61575 & 64268 & 67027 & 69754 & 72433 & 74998 & 77447 & 79744 & 81915 \\
\hline \multirow{4}{*}{$65+$} & Moderada & 39997 & 40567 & 41180 & 41821 & 42441 & 43043 & 43593 & 44103 & 44559 & 44986 & 45367 \\
\hline & Severa & 19878 & 20379 & 20924 & 21497 & 22064 & 22625 & 23146 & 23635 & 24081 & 24508 & 24889 \\
\hline & G. Dependencia & 37496 & 38450 & 39479 & 40542 & 41603 & 42633 & 43611 & 44547 & 45426 & 46270 & 47054 \\
\hline & Total & 97371 & 99396 & 101584 & 103860 & 106108 & 108301 & 110350 & 112285 & 114066 & 115764 & 117310 \\
\hline
\end{tabular}

Fuente: Elaboración propia. 


\section{Cuadro 5}

Población Dependiente de 65 y más años (ambos sexos) potencial demandante del servicio de Teleasistencia según Grados de Dependencia.

Comunidad Autónoma de Castilla y León (2005-2015). Escenario 1

\begin{tabular}{|c|c|c|c|c|c|c|c|c|c|c|c|c|}
\hline \multicolumn{13}{|c|}{ Tasas de Discapacidad y Tasas de Dependencia constantes } \\
\hline $\begin{array}{l}\text { Grupos } \\
\text { de Edad }\end{array}$ & $\begin{array}{c}\text { Grados de } \\
\text { Dependencia }\end{array}$ & 2005 & 2006 & 2007 & 2008 & 2009 & 2010 & 2011 & 2012 & 2013 & 2014 & 2015 \\
\hline \multirow{4}{*}{65 a 79} & Moderada & 20552 & 20326 & 20098 & 19888 & 19638 & 19386 & 19094 & 18803 & 18495 & 18219 & 17934 \\
\hline & Severa & 5337 & 5298 & 5250 & 5198 & 5131 & 5062 & 4975 & 4881 & 4776 & 4680 & 4571 \\
\hline & G. Dependencia & 1969 & 1955 & 1937 & 1916 & 1890 & 1862 & 1828 & 1796 & 1763 & 1734 & 1705 \\
\hline & Total & 27858 & 27579 & 27285 & 27002 & 26659 & 26310 & 25897 & 25480 & 25034 & 24633 & 24210 \\
\hline \multirow{4}{*}{$80+$} & Moderada & 19445 & 20241 & 21082 & 21933 & 22804 & 23657 & 24499 & 25300 & 26064 & 26767 & 27433 \\
\hline & Severa & 6381 & 6657 & 6963 & 7283 & 7612 & 7938 & 8257 & 8563 & 8856 & 9134 & 9397 \\
\hline & G. Dependencia & 1462 & 1525 & 1594 & 1666 & 1740 & 1813 & 1885 & 1954 & 2020 & 2082 & 2141 \\
\hline & Total & 27288 & 28423 & 29639 & 30882 & 32155 & 33407 & 34641 & 35818 & 36941 & 37984 & 38971 \\
\hline \multirow{4}{*}{$65+$} & Moderada & 39997 & 40567 & 41180 & 41821 & 42441 & 43043 & 43593 & 44103 & 44559 & 44986 & 45367 \\
\hline & Severa & 11718 & 11955 & 12212 & 12481 & 12742 & 13000 & 13231 & 13445 & 13633 & 13814 & 13968 \\
\hline & G. Dependencia & 3431 & 3480 & 3531 & 3582 & 3630 & 3675 & 3714 & 3751 & 3784 & 3817 & 3846 \\
\hline & Total & 55147 & 56002 & 56923 & 57885 & 58814 & 59717 & 60538 & 61298 & 61975 & 62617 & 63181 \\
\hline
\end{tabular}

Fuente: Elaboración propia.

\section{EL MODELO DE COLAS}

La Teoría de Colas es una rama de la Investigación Operativa que estudia el comportamiento de los sistemas de atención a los clientes que demandan servicio y en los que, en determinadas ocasiones, éstos tienen que esperar para ser atendidos. Proporciona modelos matemáticos que se utilizan para analizar los diferentes tipos de sistemas en los que los clientes que demandan servicio deben esperar para ser atendidos (Figura 1). Estos modelos son muy útiles para estudiar cómo debe funcionar un sistema de colas o de líneas de espera, de tal forma, que éste sea lo más eficiente posible en términos de costes (Escudero, 1972). Sin embargo, hay que tener en cuenta que una excesiva capacidad de servicio lleva consigo costos elevados. Por tanto, los modelos de colas permiten establecer un equilibrio apropiado entre los costes y la calidad del servicio que se ofrece a los clientes. Son muchas las situaciones de la vida diaria de cualquier persona que pueden ser analizadas a través de los modelos de colas, tales como la prestación de servicios sociales y, entre ellos, el servicio de Teleasistencia. 
Figura 1

Servicio de Teleasistencia

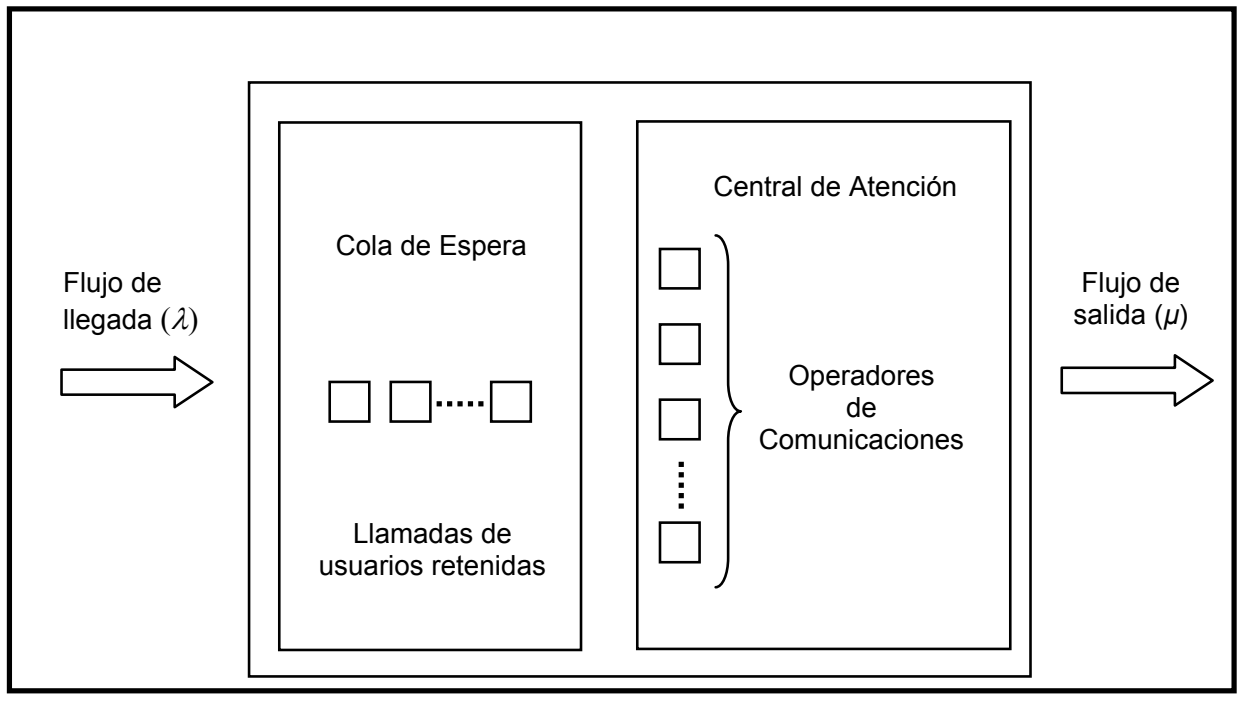

Fuente: Elaboración propia.

Tener que esperar a ser atendido es una pérdida de tiempo, lo que hace que sea considerado por las personas como un factor de calidad de vida y para un país como un factor de eficiencia de su economía. Los retrasos en los servicios pueden generar pérdidas en los negocios e ineficiencias y mala imagen en las organizaciones. Del mismo modo, se considera que el número de operadores que atienden las llamadas es independiente del número de usuarios que llaman al servicio de Teleasistencia ${ }^{12}$.

Para analizar el servicio de Teleasistencia a través del modelo de colas es preciso conocer (o estimar) la distribución de probabilidad que siguen los tiempos entre las llegadas y los tiempos entre las salidas del sistema. Cuando se desconocen los valores de alguna de estas variables, se utilizan técnicas de simulación. La secuencia metodológica seguida por los modelos de la Teoría de Colas es la siguiente: motivación de la aparición de la cola de espera en el servicio o en el sistema; deducción de las fórmulas del modelo de colas consi-

${ }^{12}$ Cuando el número de servidores (operadores de comunicaciones) es independiente del número de personas (usuarios) que demandan atención a un determinado servicio, se dice que es un sistema de Poisson Especializado. 
derado; y planteamiento y obtención de los valores de las variables y parámetros de estos modelos ${ }^{13}$.

\subsection{Variables y parámetros del modelo}

Para realizar el análisis del servicio de Teleasistencia utilizamos un modelo de colas del tipo $\mathrm{M} / \mathrm{M} / \mathrm{C} / \mathrm{K}$, con $\mathrm{K}>\mathrm{C}$. Las variables y parámetros que caracterizan a dicho modelo pueden clasificarse como indicadores de diseño, indicadores de gestión o desempeño de interés tanto para el administrador como para el usuario (Kaplan y Norton, 1996; Najar y Rajan, 2005; Singer y Donoso, 2007; Singer et al., 2008), y son las siguientes:

\section{Indicadores de diseño:}

$\tau_{\mathrm{i}}$ : Tiempo que transcurre entre la llegada del $\mathrm{i}-1$ cliente y el cliente $\mathrm{i}$, con $\mathrm{i}=1$, $2, \ldots$ Como estos tiempos se consideran variables aleatorias independientes e igualmente distribuidas (v.a.i.i.d.), se denota por $\tau$ al tiempo entre llegadas al servicio.

$\mathbf{s}_{\mathbf{i}}$ : Tiempo que transcurre desde el inicio del servicio al cliente i-ésimo hasta su terminación, con $\mathrm{i}=1,2, \ldots \mathrm{Al}$ igual que para los tiempos entre llegadas, se supone que éstos son v.a.i.i.d. y, por tanto, se va a denotar por $\mathrm{s}$ al tiempo transcurrido entre servicios.

$\lambda$ : Número esperado (medio) de usuarios que llaman al Servicio de Teleasistencia demandando atención en la unidad de tiempo. Denota el tanto o tasa de llamada al sistema y se define por la expresión $\lambda=1 / \mathrm{E}(\tau)$, siendo $\mathrm{E}(\tau)$ el tiempo medio que tarda un usuario en llamar al servicio.

$\lambda_{\mathrm{a}}$ : Número medio de usuarios que llaman y entran en el Servicio de Teleasistencia demandando atención en la unidad de tiempo. Denota el tanto real de llamada al sistema para modelos de colas con capacidad limitada y se define por la expresión $\lambda_{\mathrm{a}}=\lambda \cdot\left(1-\mathrm{P}_{\mathrm{k}}\right)$, con $\mathrm{P}_{\mathrm{k}}$ la probabilidad de que haya $\mathrm{k}$ usuarios en el servicio en cualquier momento.

${ }^{13}$ La nomenclatura que describe los modelos de colas viene establecida por la notación de Kendall $\mathrm{M} / \mathrm{M} / \mathrm{C} / \mathrm{K} / \mathrm{m} / \mathrm{z}$. Así, la letra $\mathrm{M}$ de la primera casilla denota que los tiempos medios entre llamadas al servicio de los usuarios se distribuyen según una ley exponencial ; la letra $\mathrm{M}$ de la segunda casilla describe que los tiempos medios de atención a las personas que demandan este tipo de servicio también siguen una ley exponencial; $C$ representa el número de operadores de comunicaciones que atienden a los usuarios que llaman al Servicio de Teleasistencia; K la capacidad del servicio que, en este caso, es limitada y mayor que el número de operadores que prestan atención a las personas que lo demandan; m, el tamaño de la población potencial que tiene acceso al servicio y z, la prioridad de atención de los usuarios en el servicio (Taha, 1997; Hilier et al., 2001). 
$\mu$ : Número esperado (medio) de usuarios que atiende dicho servicio por unidad de tiempo. Representa el tanto o tasa de atención al usuario o persona dependiente y se define como $\mu=1 / \mathrm{E}(\mathrm{s})$, con $\mathrm{E}(\mathrm{s})$ el tiempo medio que tarda un usuario cualquiera en ser atendido por un operador de comunicaciones del servicio de Teleasistencia.

C: Número de operadores de comunicaciones que atienden las llamadas de los usuarios al servicio de Teleasistencia.

\section{Indicadores de desempeño de interés para el administrador:}

$\rho$ :Probabilidad de estar ocupado un operador de comunicaciones. Se denomina factor de utilización o intensidad de tráfico del sistema analizado, en este caso, el servio de teleasistencia y mide también el porcentaje de tiempo ocupado por unidad de tiempo de cada operador: $\rho=\lambda / \mathrm{C} \cdot \mu$.

$\rho_{\mathrm{a}}$ : Probabilidad de estar ocupado un operador de comunicaciones cuando el servicio de Teleasistencia tiene capacidad limitada. Representa también el porcentaje real que el operador de comunicaciones está ocupado en cada unidad de tiempo: $\rho_{\mathrm{a}}=\lambda_{\mathrm{a}} / \mathrm{C} \cdot \mu$.

$\mathbf{L}_{\mathbf{q}}$ : Número medio de usuarios en la cola en espera de ser atendidos en cualquier momento.

$$
L_{q}=\left\{\begin{array}{l}
\frac{P_{C} \cdot \rho}{(1-\rho)^{2}} \cdot\left(1-\rho^{k-C}-(K-C) \cdot \rho^{k-C+1}\right), \text { si } \rho \neq 1 \\
\frac{(K-C+1) \cdot(K-C)}{2} \cdot P_{C}, \rho=1 .
\end{array}\right.
$$

$\mathbf{L}_{\mathbf{s}}$ : Número medio de usuarios que están siendo atendidos por los operadores del servicio de Teleasistencia en cualquier momento: $\mathrm{L}_{\mathrm{s}}=\mathrm{C} \rho \cdot\left(1-\mathrm{P}_{\mathrm{k}}\right)$.

$\mathbf{L}$ : Número medio de usuarios en el servicio de Teleasistencia en cualquier momento, es decir, los que están esperando a ser atendidos más los que están siendo atendidos: $\mathrm{L}=\mathrm{L}_{\mathrm{q}}+\mathrm{L}_{\mathrm{s}}$.

\section{Indicadores de desempeño de interés para el usuario:}

$\mathbf{W}_{\mathbf{q}}$ : Tiempo medio que el usuario está en la línea de espera hasta que es atendido por el operador de comunicaciones. $\mathrm{W}_{\mathrm{q}}=\mathrm{L}_{\mathrm{q}} / \lambda_{\mathrm{a}}$. 
W : Tiempo medio que el usuario está en el servicio de Teleasistencia hasta que se va del mismo. $\mathrm{W}=\mathrm{L} / \lambda_{\mathrm{a}}$.

$\mathbf{P}_{0}$ : Probabilidad de que en el servicio de Teleasistencia no haya ningún usuario demandando atención en cualquier momento de tiempo.

$$
\mathrm{P}_{0}=\left\{\begin{array}{l}
\rho^{\mathrm{n}} \cdot \frac{1-\rho^{\mathrm{k}+1}}{1-\rho}, \text { si } \rho \neq 1 \\
\frac{1}{(1+\mathrm{K})}, \text { si } \rho=1 .
\end{array}\right.
$$$$
P_{0}=\left(\sum_{c=0}^{c-1} \frac{(c \rho)^{n}}{n !}+\frac{(c \rho)^{c}}{c !(1-\rho)}\right)^{-1} \text {, si } \rho \neq 1 .
$$

$\mathbf{P}_{\mathbf{n}}$ :Probabilidad de que en el servicio de Teleasistencia haya $\mathrm{n}$ usuarios demandando atención en cualquier momento de tiempo.

$$
P_{n}=\left\{\begin{array}{l}
\rho^{n} \frac{1-\rho}{1-\rho^{k+1}}, \text { si } \rho \neq 1 ; \rho=1 \\
\frac{1}{(1+K)}, \text { si } \rho=1 .
\end{array}\right.
$$

\subsection{Hipótesis del modelo}

Para proceder a analizar los costes derivados del servicio de Teleasistencia en Castilla y León a través del modelo de colas planteado, es necesario especificar diversas hipótesis:

- Se considera que el servicio de Teleasistencia tiene capacidad limitada; es decir, que cuando el número de llamadas exceda al número de operadores de comunicaciones que las atienden, los usuarios han de esperar; que los potenciales usuarios que pueden demandar dicho servicio son ilimitados; que el primer usuario en llamar es el primero en ser atendido; y que la capacidad del servicio es mayor que el número de operadores que atienden a los usuarios; y por lo tanto, que consideramos un modelo de colas del tipo $\mathrm{M} / \mathrm{M} / \mathrm{C} / \mathrm{K}$.

- Se tiene en cuenta para este análisis, que una persona con dependencia moderada demanda 1 hora de Servicio de Ayuda a Domicilio (SAD); una persona con dependencia severa, 3 horas; y una persona con gran dependencia, 5 horas de SAD. En la Cuadro 6, se describen los costes medios, en euros/hora, para el servicio de Teleasistencia y para el Servicio de Ayuda a Domicilio ${ }^{14}$ según las hipótesis establecidas para su cálculo.

${ }^{14}$ El cálculo de estos costes se ha realizado a partir de los datos recogidos en el Informe de las Personas Mayores en España (IMSERSO, 2008). Cuando se demanda atención al servicio de 


\section{Cuadro 6}

Costes medios (euros/hora) de Servicios de Atención por persona

dependiente en Castilla y León (2005-2015)

\begin{tabular}{|l|c|}
\hline Tipos de Servicios de Atención & Coste Público \\
\hline Servicio de Teleasistencia & $0,03(€ /$ hora $)$ \\
\hline Servicio de Ayuda a Domicilio & $13,88(€ / \mathrm{hora})$ \\
\hline
\end{tabular}

Fuente: Elaboración propia.

- Los costes por usuario que se generan en el servicio de Teleasistencia en dicha región por la demanda de atención de las personas con diferentes grados de dependencia que éstas pueden presentar, se describen en la Cuadro 7.

\section{Cuadro 7}

Hipótesis sobre Costes por usuario (euros/hora) del servicio de Teleasistencia según Grados de Dependencia

\begin{tabular}{|l|c|c|c|}
\hline $\begin{array}{l}\text { Grados Dependencia/ } \\
\text { Costes }\end{array}$ & Dependencia Moderada & $\begin{array}{c}\text { Dependencia } \\
\text { Severa }\end{array}$ & Gran Dependencia \\
\hline Coste pérdida usuario & $13,88 € / \mathrm{h}$. & $41,64 € / \mathrm{h}$. & $69,40 € / \mathrm{h}$. \\
\hline Coste de espera & $3,47 € / \mathrm{h}$. & $10,41 € / \mathrm{h}$. & $17,35 € / \mathrm{h}$. \\
\hline Coste del servidor & $10,65 € / \mathrm{h}$. & $10,65 € / \mathrm{h}$. & $10,65 € / \mathrm{h}$. \\
\hline Coste de servicio & $0,03 € / \mathrm{h}$. & $0,03 € / \mathrm{h}$. & $0,03 € / \mathrm{h}$. \\
\hline
\end{tabular}

Fuente: Elaboración propia.

- La penalización por usuario perdido se valora en término de horas de SAD.

- Los costes totales de atención a los usuarios según los diferentes grados de dependencia para el período temporal analizado se consideran constantes.

- El número medio de llamadas por hora a la central de atención del servicio de Teleasistencia de la región se distribuyen según una ley exponencial y se estima en 120, de acuerdo con la información suministrada por la entidad gestora.

Teleasistencia y no existen operadores disponibles, se genera una penalización para la compañía que gestiona el servicio y cuya cuantificación se calcula en horas de SAD equivalentes según el grado de dependencia que presente el usuario. Esta penalización por usuario perdido constituye un coste adicional para dicho servicio. 
- Según datos recogidos de la misma fuente, se estima que el tiempo medio de atención a cada persona dependiente que llama a dicho servicio es de 5 minutos y se supone, igualmente, una variable aleatoria que se distribuye de forma exponencial. Por consiguiente, el tanto medio de atención del servicio es de 12 usuarios por hora.

\subsection{Datos del modelo}

Para estimar los costes totales que se derivan de la prestación del servicio de Teleasistencia en la comunidad Autónoma de Castilla y León, es necesario partir de una serie de datos que van a posibilitar la simulación del modelo de colas (Pazos et al., 2003). Concretamente, se dispone de la siguiente información que ha sido facilitada por la entidad gestora de dicho servicio en la región y por la Gerencia de Servicios Sociales de Castilla y León:

- La central de atención telefónica dispone de 28 líneas operativas a las que se pasan las llamadas realizadas por las personas dependientes ${ }^{15}$, y de 40 operadores de comunicaciones fijos, además de 9 personas de refuerzo para atender las llamadas durante los tres turnos del día, es decir, que en cada turno trabajan 13 operadores de media. Por tanto, se supone que se trabaja con un modelo de colas con capacidad limitada, siendo $\mathrm{K}=28$.

- El coste de espera de cada persona con dependencia que llama al servicio de Teleasistencia y que no puede ser atendida por la central de atención equivale a la cuarta parte del coste medio por usuario perdido y alcanza los siguientes valores: $3,47 € /$ hora por persona con dependencia moderada; $10,41 € /$ hora por persona con dependencia severa; $17,35 € /$ hora por persona con gran dependencia. Cuando se consideran todos los grados de dependencia de forma conjunta, dicho coste será de 5,83 €/hora por persona que demanda atención ${ }^{16}$.

- El coste de cada operador de comunicaciones viene establecido por su salario de acuerdo con la Tabla Salarial revisada según el Convenio del Sector de Oficinas y Despachos y que asciende a $1.700 € /$ mes $(8$ horas de trabajo a jornada completa), equivalente a 7,1 €/hora. Para realizar un estudio más ajustado a la realidad, se considera que otra persona adicional

${ }^{15}$ Cuando todas las líneas telefónicas están en uso las llamadas no son atendidas, dando tono de ocupado. Los demandantes de este servicio desean atención inmediata y no tener que intentarlo de nuevo, por lo que es importante establecer un número adecuado de líneas disponibles que contribuya a lograr este objetivo a un coste razonable.

${ }^{16}$ Este coste está calculado como el cociente entre la suma ponderada de los costes por usuario perdido según su grado de dependencia $(71,8 \%$ población con dependencia moderada; $22,1 \%$ con dependencia severa y el $6,1 \%$ en situación de gran dependencia) y el total de personas dependientes para todos los grados que cada año pueden demandar dicho servicio. 
realiza esta tarea de forma voluntaria durante la mitad de la jornada labo$\mathrm{ral}^{17}$. De esta manera, al anterior salario se le suma $850 € /$ mes (4 horas de trabajo/día), equivalente a $3,55 € /$ hora. Por tanto, el coste salarial por operador es de $10,65 € /$ hora.

- El coste de servicio de cada operador de comunicaciones se obtiene del Informe del Servicio de Atención a Domicilio de la Gerencia de Servicios Sociales de la Comunidad Autónoma de Castilla y León (IMSERSO, 2008), y asciende a $273,96 € /$ año/usuario, es decir, $0,03 € /$ hora/usuario y se supone constante para todo el período del análisis. Este coste de servicio incluye los gastos generados por la utilización del teléfono, el mobiliario y los inmuebles que son necesarios para atender a las personas dependientes que llaman demandado atención al servicio de Teleasistencia.

- El coste por pérdida de usuario ocasionado por la capacidad limitada de la central de atención, se estima equivalente a 1 hora de $\operatorname{SAD}(13,88 €)$ para las personas con dependencia moderada; a 3 horas de SAD $(41,64 €)$ para las personas con dependencia severa; y a 5 horas de $\operatorname{SAD}(69,40 €)$ para las personas con gran dependencia (Cuadro 6). El coste medio por usuario cuando se considera de forma conjunta todas las personas sin hacer distinción del grado es de 23,33 €/hora.

\subsection{Resultados de la simulación del modelo}

A partir de las hipótesis y datos anteriormente señalados, se procede a la simulación del modelo de colas a través del programa informático WinQSB 1.0. Los resultados obtenidos se presentan a continuación tanto en términos de costes totales por usuario, como de costes agregados asociados a la prestación del servicio (Cuadros 8 y 9). El Cuadro 8 recoge los valores de las principales variables que caracterizan al modelo y el número de operadores de comunicaciones $\left(\mathrm{C}^{*}\right)$ que minimizan los costes totales por usuario. En el Cuadro 9 se describen los costes agregados diferenciados según grado de dependencia y conjuntamente para todos los grados, teniendo en cuenta el número óptimo de operadores de comunicaciones.

Las trayectorias temporales que describen los costes totales para el período 2005-2015 se representan en la Figura 2 y en la Figura 3. Así, en la Figura 2 se recogen los costes totales por usuario según grado de dependencia (moderada, severa y gran dependencia) y, en la Figura 3, se representan los costes totales agregados para todos los grados de dependencia.

${ }^{17}$ De acuerdo con ello, el coste real del servicio debe incluir el trabajo voluntario y no voluntario, aunque para la entidad gestora el trabajo voluntario no represente un compromiso de pago, es decir, un desembolso monetario. 


\section{Cuadro 8}

Número de Operadores de Comunicaciones que minimizan los Costes Totales por usuario del servicio de Teleasistencia para la Población Dependiente (ambos sexos) de 65 y más años según Grados de Dependencia. Comunidad Autónoma de Castilla y León

\begin{tabular}{|c|c|c|c|c|c|c|c|c|c|c|c|}
\hline \multicolumn{12}{|c|}{ Modelo de Colas $M / M / C / K$ y parámetros $\lambda=120 ; \mu=12 ; K=28$. } \\
\hline C & $\lambda_{\mathrm{a}}$ & $\rho_{\mathrm{a}}$ & $\mathbf{L}$ & $\mathbf{L}_{\mathbf{q}}$ & $\mathbf{W}$ & $\mathbf{W}_{\mathbf{q}}$ & $\begin{array}{l}\text { Coste del } \\
\text { Servidor }\end{array}$ & $\begin{array}{l}\text { Coste de } \\
\text { Espera }\end{array}$ & $\begin{array}{l}\text { Coste de } \\
\text { Servicio }\end{array}$ & $\begin{array}{c}\text { Coste Pérdida } \\
\text { Usuario }\end{array}$ & $\begin{array}{l}\text { Coste } \\
\text { Total }\end{array}$ \\
\hline \multicolumn{12}{|c|}{ Dependencia Moderada (DM) } \\
\hline 11 & 118,3219 & 0,8964 & 13,7197 & 3,8595 & 0,1160 & 0,0326 & 117,1500 & 13,3925 & 0,2958 & 23,1929 & 154,1302 \\
\hline 12 & 119,5038 & 0,8299 & 11,7972 & 1,8386 & 0,0987 & 0,0154 & 127,8000 & 6,3798 & 0,2988 & 6,8869 & 141,3635 \\
\hline 13 & 119,8450 & 0,7682 & 10,8588 & 0,8718 & 0,0906 & 0,0073 & 138,4500 & 3,0250 & 0,2996 & 2,1514 & 143,9260 \\
\hline \multicolumn{12}{|c|}{ Dependencia Severa (DS) } \\
\hline 12 & 119,5038 & 0,8299 & 11,7972 & 1,8386 & 0,0987 & 0,0154 & 127,8000 & 19,1394 & 0,2988 & 20,6608 & 167,8990 \\
\hline 13 & 119,8450 & 0,7682 & 10,8588 & 0,8718 & 0,0906 & 0,0073 & 138,4500 & 9,0750 & 0,2996 & 6,4541 & 154,2787 \\
\hline 14 & 119,9462 & 0,7140 & 10,4117 & 0,4162 & 0,0868 & 0,0035 & 149,1000 & 4,3327 & 0,2999 & 2,2397 & 155,9723 \\
\hline \multicolumn{12}{|c|}{ Gran Dependencia (GD) } \\
\hline 13 & 119,8450 & 0,7682 & 10,8588 & 0,8718 & 0,0906 & 0,0073 & 138,4500 & 15,1250 & 0,2996 & 10,7569 & 164,6315 \\
\hline 14 & 119,9462 & 0,7140 & 10,4117 & 0,4162 & 0,0868 & 0,0035 & 149,1000 & 7,2212 & 0,2999 & 3,7328 & 160,3539 \\
\hline 15 & 119,9790 & 0,6666 & 10,1968 & 0,1986 & 0,0850 & 0,0017 & 159,7500 & 3,4450 & 0,3000 & 1,4561 & 164,9510 \\
\hline \multicolumn{12}{|c|}{ Todos los Grados de Dependencia } \\
\hline 12 & 119,5038 & 0,8299 & 11,7972 & 1,8386 & 0,0987 & 0,0154 & 127,8000 & 16,9078 & 0,2988 & 11,5758 & 150,3934 \\
\hline 13 & 119,8450 & 0,7682 & 10,8588 & 0,8718 & 0,0906 & 0,0073 & 138,4500 & 5,0823 & 0,2996 & 3,6161 & 147,4481 \\
\hline 14 & 119,9462 & 0,7140 & 10,4117 & 0,4162 & 0,0868 & 0,0035 & 149,1000 & 2,4265 & 0,2999 & 1,2549 & 153,0812 \\
\hline
\end{tabular}

Fuente: Elaboración propia.

\section{Cuadro 9}

Costes Agregados (euros/hora) del servicio de Teleasistencia para la Población Dependiente (ambos sexos) de 65 y más años según Grados de Dependencia. Comunidad Autónoma de Castilla y León (2005- 2015)

\begin{tabular}{|c|c|c|c|c|c|c|}
\hline Años/Grados de Dependencia & 2005 & 2007 & 2009 & 2011 & 2013 & 2015 \\
\hline \multicolumn{7}{|c|}{ Modelo de Colas $M / M / C / K$ con parámetros $\lambda=120 ; \mu=12 ; C^{*}=12 ; K=28$} \\
\hline Dependencia Moderada & 5654116 & 5821349 & 5999608 & 6162459 & 6299016 & \\
\hline \multicolumn{7}{|c|}{ Modelo de Colas $M / M / C / K$ con parámetros $\lambda=120 ; \mu=12 ; C^{*}=13 ; K=28$} \\
\hline Dependencia Severa & 1807838 & 1884051 & 1965819 & 2041261 & 2103282 & 215496 \\
\hline \multicolumn{7}{|c|}{ Modelo de Colas $M / M / C / K$ con parámetros $\lambda=120 ; \mu=12 ; C^{*}=14 ; K=28$} \\
\hline Gran Dependencia & 550174 & 566210 & 582085 & 595554 & 606779 & 616721 \\
\hline Total (MTGD) & 8012128 & 8271610 & 8547512 & 8799275 & 9009077 & 9184924 \\
\hline \multicolumn{7}{|c|}{ Modelo de Colas $M / M / C / K$ con parámetros $\lambda=120 ; \mu=12 ; C^{*}=13 ; K=28$} \\
\hline odos los Grados de Dependencia (TGD) & 8131320 & 8393188 & 8672013 & 8926213 & 9138096 & 9184924 \\
\hline
\end{tabular}

Fuente: Elaboración propia. 
Figura 2

Costes totales por usuario para cada grado de dependencia

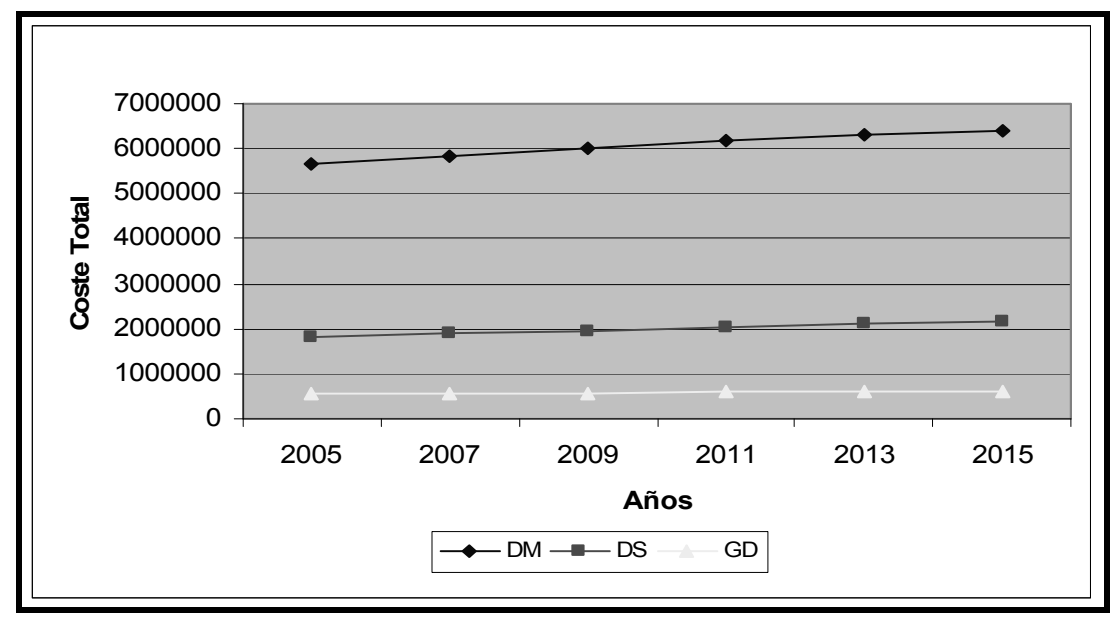

Fuente: Elaboración propia.

Figura 3

Costes agregados para todos los grados de dependencia

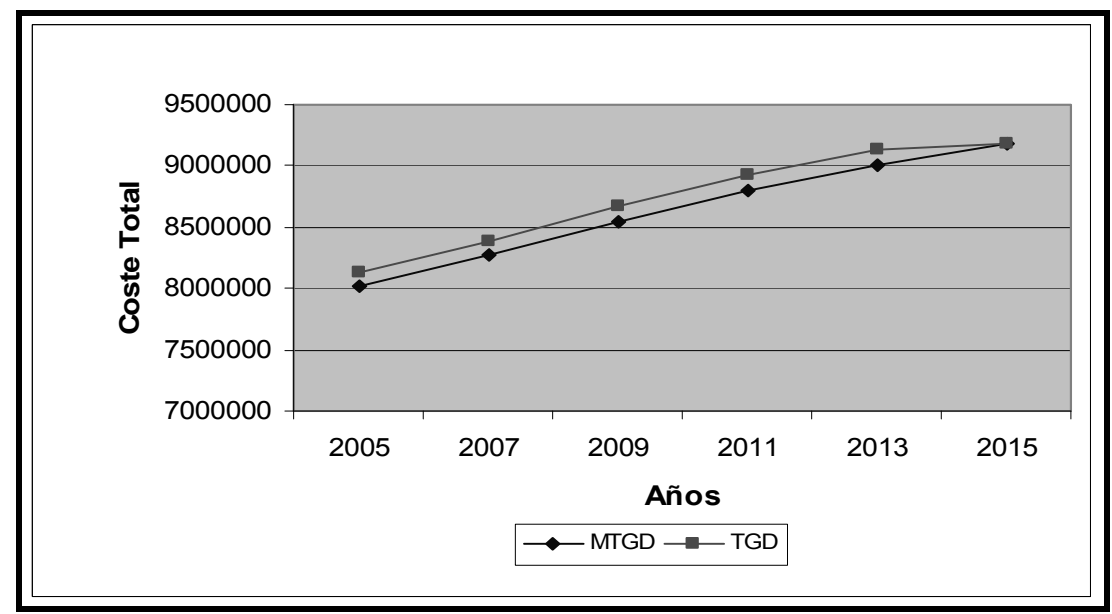

Fuente: Elaboración propia.

\section{ANÁLISIS DE SENSIBILIDAD DEL MODELO}

A continuación, se realiza un análisis de sensibilidad del modelo con el fin de poder comprobar los valores que toma alguna de las variables más significativas del mismo (en concreto, el coste total por persona atendida) ante pequeñas variaciones en algunos de sus parámetros, tales como el número de opera- 
dores de comunicaciones; el tanto de llamada del usuario $(\lambda)$; el tanto de atención al usuario $(\mu)$; y la capacidad del servicio $(\mathrm{K})$, caeteris páribus, manteniendo constantes los demás parámetros.

Los valores obtenidos del análisis de sensibilidad del modelo de colas para la variable más relevante, el coste total por usuario atendido, cuando se considera de forma conjunta todos los grados de dependencia se recogen a continuación (Figuras 4, 5, 6 y 7).

En la Figura 4 se presentan los valores que alcanza el coste total por persona atendida ante variaciones en el tanto de llamada del usuario, considerando el número de operadores que optimizan el coste total cuando se toman conjuntamente todos los grados de dependencia. Como se puede comprobar, dicho coste crece a medida que lo hace el tanto de llamada $(\lambda)$ y de forma más rápida a partir de las 120 llamadas por hora.

Figura 4

Coste Total ante variaciones en el tanto de llegada

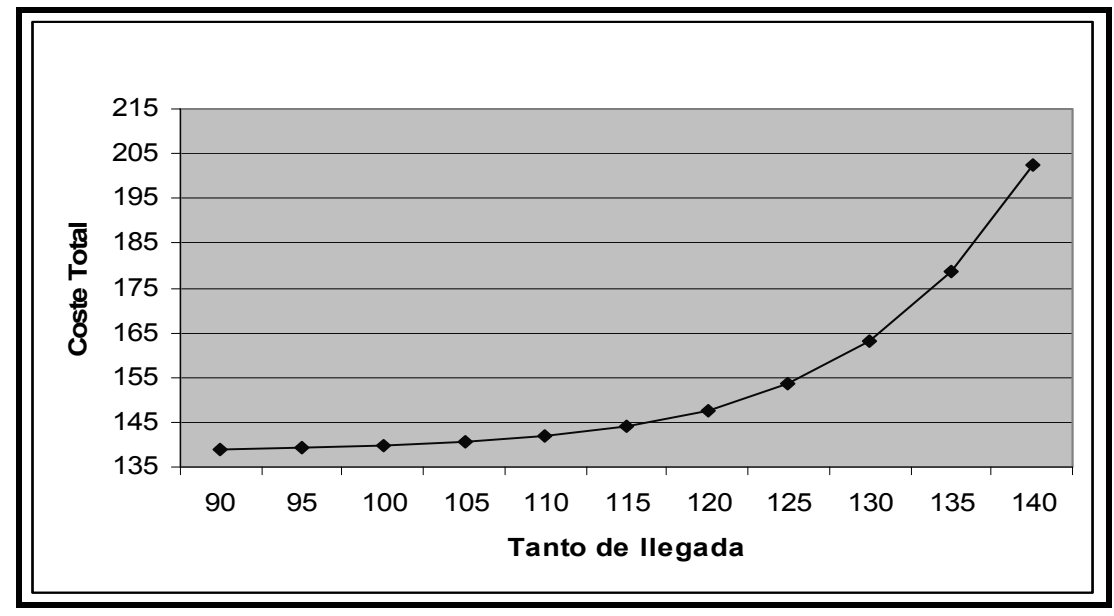

Fuente: Elaboración propia.

En la Figura 5 se constata que el coste total por persona atendida decrece a medida que crece el tanto de atención al usuario $(\mu)$. En este caso, tienen un comportamiento opuesto, es decir, si una variable aumenta la otra disminuye y viceversa. Una vez alcanzado los 14 usuarios en promedio por hora atendidos, dicho coste se mantiene constante. 
Figura 5

Coste Total ante variaciones en el tanto de servicio (atención)

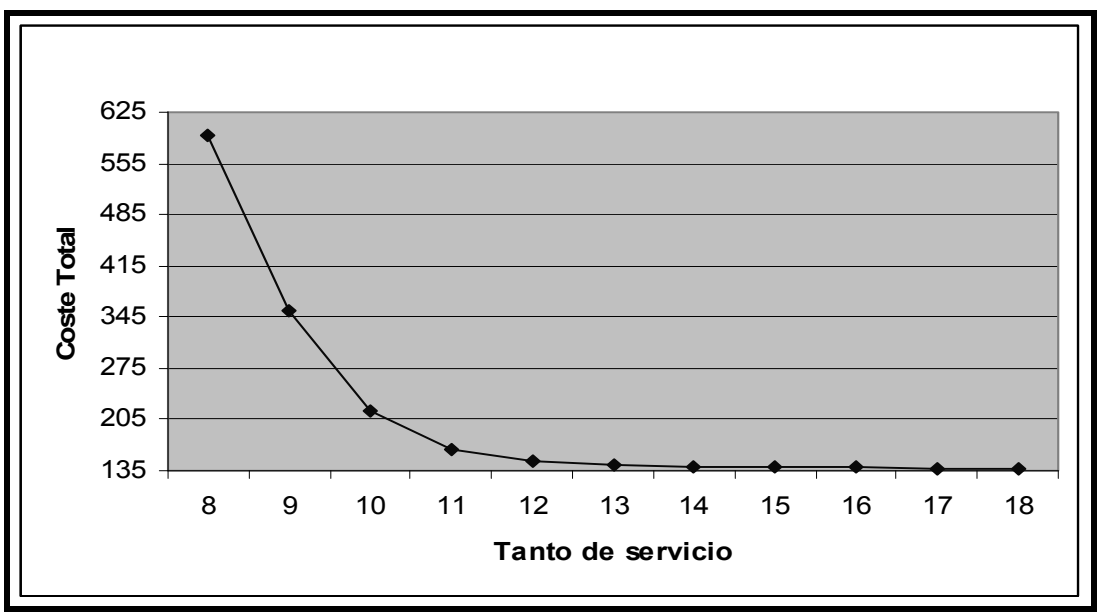

Fuente: Elaboración propia.

La Figura 6 recoge los valores del coste total por persona atendida al variar la capacidad del servicio(K), constatando que estas variables evolucionan de forma inversa. La disminución que experimenta este coste a medida que crece la capacidad de servicio es poco significativa, lo que hace que la eficiencia del servicio depende muy poco de las líneas telefónicas disponibles.

Figura 6

Coste Total ante variaciones en la capacidad del servicio

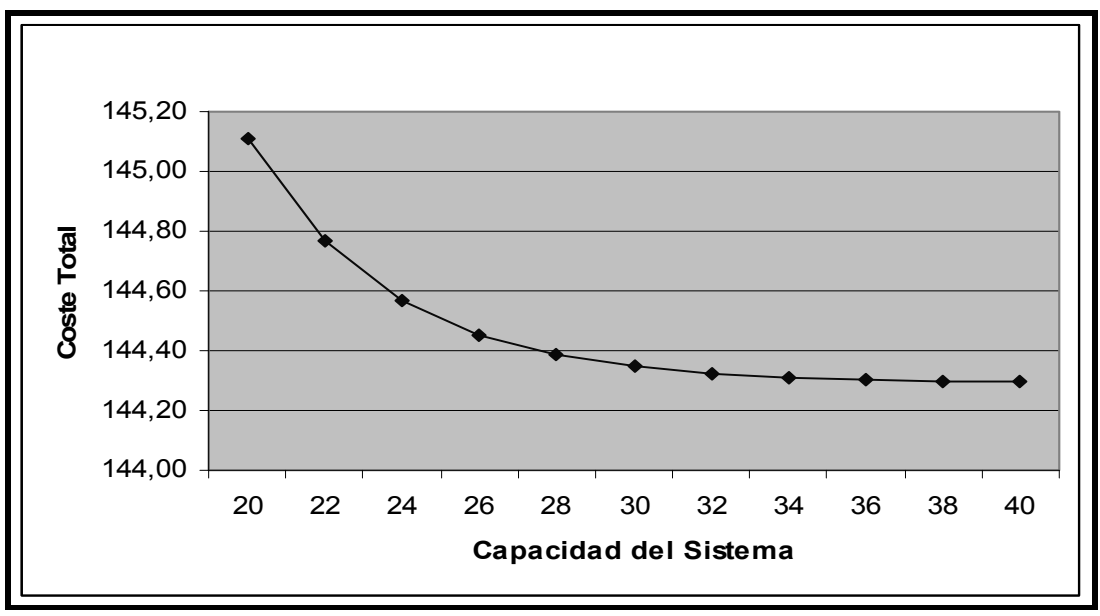

Fuente: Elaboración propia. 
La Figura 7 muestra cómo varía el coste total por persona atendida ante variaciones en el número de operadores de comunicaciones que atienden al servicio de Teleasistencia. Se comprueba que a medida que aumenta el número de operadores, el coste total por persona atendida se reduce y alcanza su mínimo cuando el número de operadores es de $13\left(\mathrm{C}^{*}=13\right)$. A partir de este valor, dicho coste comienza a crecer de nuevo a medida que el número de operadores aumenta.

Figura 7

Coste Total ante variaciones en el número de operadores del servicio

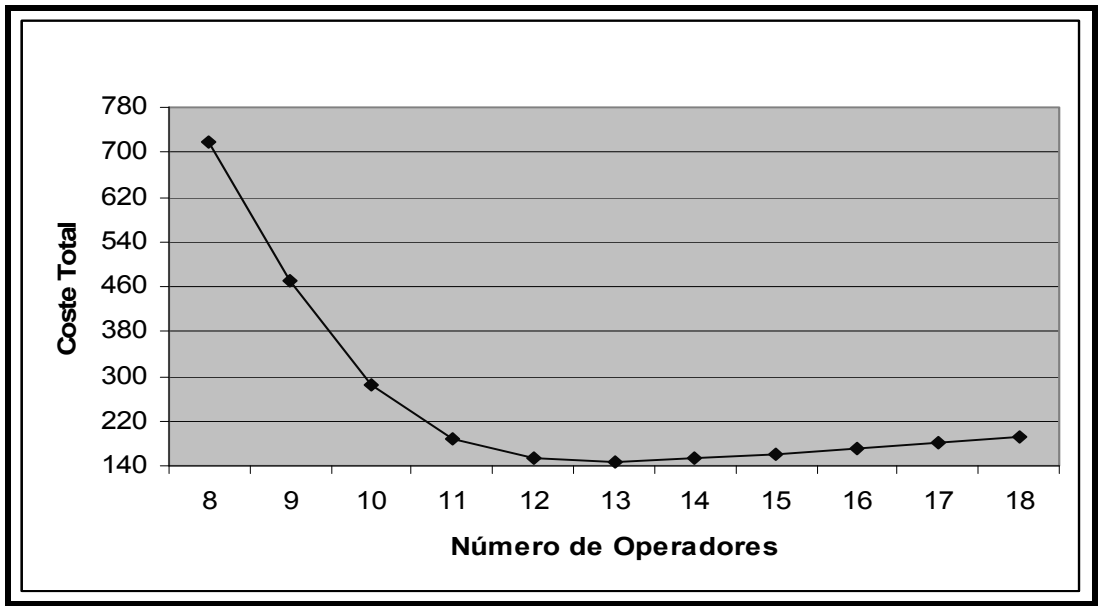

Fuente: Elaboración propia.

\section{CONCLUSIONES}

En este trabajo se ha tratado de analizar la eficiencia del servicio de Teleasistencia que atiende a las personas ancianas dependientes desde la perspectiva de los costes económicos que optimizan su funcionamiento, tomando como referencia empírica la situación de la Comunidad Autónoma de Castilla y León proyectada para el período 2005-2015. Para ello, se ha planteado un modelo matemático fundamentado en la Teoría de Colas, considerando diversas hipótesis de comportamiento en los costes y en la gestión del servicio. A partir de datos suministrados por la entidad gestora del citado servicio en la región (Cruz Roja Española) y de otros procedentes de la propia Administración Regional (Gerencia de Servicios Sociales de Castilla y León), se ha procedido a realizar la simulación del modelo planteado. 
Los resultados obtenidos de dicha simulación ponen de manifiesto que el número óptimo de operadores de comunicaciones $\left(\mathrm{C}^{*}\right)$ que minimiza los costes totales por usuario del servicio de Teleasistencia en la Comunidad Autónoma de Castilla y León alcanzaría, concretamente, los valores 12,13 y 14 para cada uno de los grados de dependencia considerados (moderada, severa y gran dependencia), respectivamente. En cambio, si se tienen en cuenta de forma conjunta todos los grados de dependencia, el número óptimo resultante sería de 13 operadores. Para este valor del número de operadores, los costes agregados para el conjunto de la población anciana dependiente de la región ascienden a 8.131.320 euros en el año 2005 y a 9.184 .924 euros en el 2015, lo que supone un incremento del 13\% durante el período temporal considerado. Asimismo, se constata que el coste total por usuario crece a medida que aumenta el grado de dependencia, correspondiendo los costes más elevados al caso de la atención a las personas ancianas en situación de gran dependencia.

El análisis realizado para los costes agregados (los vinculados a la prestación de este servicio para el conjunto de la población anciana dependiente potencialmente usuaria) muestra que éstos son también crecientes a lo largo del horizonte temporal considerado, en consonancia con el progresivo envejecimiento demográfico que caracteriza a dicha región. Los costes más elevados estarían asociados a la atención de las personas con dependencia moderada, mientras que los más reducidos corresponderían a las personas con gran dependencia. Por tanto, el número total de potenciales usuarios resulta ser más influyente en la trayectoria creciente de los costes agregados del servicio que el grado de dependencia que presenta el usuario del mismo, lo que hace especialmente relevante la evolución de la población anciana dependiente asociada al proceso de envejecimiento demográfico de la región.

Del análisis de sensibilidad realizado para los parámetros más significativos del modelo, se desprende que el coste total por usuario crece en proporción directa a como lo hace el tanto de llamada $(\lambda)$. No obstante, dicho coste aumenta de forma mucho rápida a partir de un determinado número medio de llamadas (120 llamadas por hora) y coincide prácticamente con el dato facilitado por la entidad gestora del servicio en la región.

También se comprueba que el coste total varía en proporción inversa a como lo hace el tanto de atención al usuario $(\mu)$. Sin embargo, una vez alcanzado el promedio de 14 usuarios por hora atendidos, dicho coste se mantiene constante. Por tanto, dicho valor medio está próximo al que corresponde con el dato real.

Del mismo modo, se constata que los costes totales por usuario son escasamente sensibles a modificaciones en la capacidad del sistema $(\mathrm{K})$; es decir, que la eficiencia del servicio depende en escasa medida del número de líneas telefó- 
nicas disponibles para la atención del usuario. En este caso, el dato real (28 líneas) aún se encuentra por debajo del valor óptimo, si bien la reducción del coste no resultaría especialmente significativa.

En cambio, el coste total por usuario sí se muestra sensible a cambios en el tanto de llamada $(\lambda)$ y especialmente sensible a variaciones en el tanto de atención al usuario $(\mu)$; es decir, que dicho coste depende en mayor grado de la duración media de la atención prestada en cada llamada que del número medio de llamadas realizadas al servicio. También se observa que el coste total por usuario disminuye hasta alcanzar el número de operadores de comunicaciones que minimizan los costes del servicio $\left(\mathrm{C}^{*}\right)$ y que, como se ha señalado, es de 13 personas. A partir de este valor, este coste crece a medida que aumenta el número de operadores.

A la vista de estos resultados cabría destacar, por un lado, que el servicio de Teleasistencia que actualmente presta Cruz Roja Española en Castilla y León opera de forma bastante eficiente, ya que el diseño de su estructura organizativa y sus recursos humanos se ajusta bastante a los resultados que, de acuerdo con el análisis que ha sido realizado siguiendo la metodología de la Teoría de Colas, conseguirían minimizar los costes totales por usuario que conlleva dicho servicio. Por otro, el análisis realizado también ha puesto de manifiesto diversos resultados que pueden resultar útiles para orientar la toma de decisiones de la entidad gestora con vistas a aumentar la eficiencia en la prestación del servicio sin perjudicar, a cambio, la calidad de la atención, como puede ser cualquier inversión en tecnología que disminuya el tiempo medio de atención al usuario.

\section{REFERENCIAS BIBLIOGRÁFICAS}

CASADO MARÍN, D. y LÓPEZ I CASASNOVAS, G. (2004): Vejez, Dependencia y Cuidados de Larga Duración. Barcelona: Fundación La Caixa.

CRUZ ROJA ESPAÑOLA (2002): "Teleasistencia Domiciliaria, un Servicio con Calidad". Documentación Social, nº 128, pp. 317-330.

ESCUDERO, L. F. (1972): Aplicaciones de la Teoría de Colas. Bilbao: Editorial Deusto.

GÓMEZ GARCÍA, J.Mª; PELÁEZ FERMOSO, F.J. y GARCÍA GONZÁLEZ, A. (2005). "Repercusiones del envejecimiento demográfico sobre el sistema público de pensiones en Castilla y León". Estudios de Economía Aplicada, $n^{\circ} 23$ (1), pp. 235-236. 
HILLIER, K. S., HILLIER, M. S. y LIEBERMAN, G. J. (2001): Métodos Cuantitativos para Administración. Un Enfoque de Modelos y Casos de Estudio con Hojas de Cálculo. México: Erwin McGraw-Hill.

IMSERSO (2005): Libro Blanco sobre la Atención a las Personas en Situación de Dependencia en España Madrid: Secretariado de Estado de Servicios Sociales, Familias y Discapacidad. Ministerio de Trabajo y Asuntos Sociales.

IMSERSO (2008): Las Personas Mayores en España. Datos Estadísticos Estables y por Comunidades Autónomas. Informe 2008. Madrid: Ministerio de Trabajo y Asuntos Sociales. Colección Documentos. Serie Documentos Estadísticos, 22019.

INE (2002): Encuesta sobre Discapacidades, Deficiencias y Estados de Salud, (EDDES, 1999). Madrid: Instituto Nacional de Estadística.

INE (2005): Proyecciones de la Población de España 2002-2017 a partir del Censo de Población de 2001. Madrid: Instituto Nacional de Estadística.

INFORME PFIZER (2002): Dependencia y Necesidades Asistenciales de los Mayores en España. Previsión al Año 2010. Madrid: Fundación Pfizer.

JIMÉNEZ LARA, A. (2004): "Perfiles de Dependencia de la Población Española y Necesidades de Cuidados de Larga Duración", En: J. Sánchez Fierro (eds): Libro Verde de la Dependencia en España. Madrid: Fundación AstraZeneca.

KAPLAN, R. y NORTON, D. (1996): The Balanced Scorecard. Boston: Harvard Business School Press.

LÓPEZ CASASNOVAS, G., COMAS HERRERA, A., MONTEVERDE VERDENELLI, M., CASADO MARÍN, D., CASO GARCÍA, J. R. y IBERN REGÁS, P. (2005): Envejecimiento y Dependencia. Situación Actual y Retos de Futuro. Barcelona: Estudios Caixa Catalunya, 2.

LÓPEZ, D. (2008): "Aproximación a la Topología de la Teoría del Actor-Red. Análisis de las Espacialidades de un servicio de Teleasistencia Domiciliaria". Revista de Antropología Iberoamericana (AIBR) n 1, pp. 113-138.

MONTEVERDE VERDENELLI, M. (2004): "Discapacidades de las Personas Mayores en España: Prevalencia, Duraciones e Impacto sobre los Costes de Larga Duración". Tesis Doctoral. Departamento de Econometría, Estadística y Economía Española. Barcelona: Universidad de Barcelona.

MORAGAS MORAGAS, R., CRISTÓFOL ALLUÉ, R. y G.I.E. (2003): El Coste de la Dependencia al Envejecer. Madrid: Editorial Herder S.L.

NAGAR, V. y RAJAN, M. V. (2005): "Measuring Costumer Relationschips: The case of the Retail BankingIndustry". Management Science, $\mathrm{n}^{\circ} 51$ (6), pp. 904-919.

OCDE (2001): "Fiscal Implications of Ageing: Projections of Age-Related Spending". OECD Economic Outlook, n 69, pp. 145-167.

PAZOS, J. J., SUÁREZ, A. y DÍAZ, R. P. (2003): Teoría de Colas y Simulación de Eventos Discretos. Madrid: Pearson Educación. 
PÉREZ BUENO, J. (2006): "La Configuración de la Autonomía Personal y la Necesidad de Apoyos Generalizados como Nuevo Derecho Social". Revista del Ministerio de Trabajo y Asuntos Sociales, ${ }^{\circ}$ 60, pp. 35-45.

PUGA GONZÁLEZ, M. D. y ABELLÁN GARCÍA, A. (2001): Dependencia y Necesidades Asistenciales de los Mayores en España. Previsión al año 2010. Madrid: Fundación Pfizer.

RODRÍGUEZ RODRÍGUEZ, P. y FERREIRO GASTÓN, E. (1997): Evaluación de los servicios de Teleasistencia Domiciliaria. Madrid: IMSERSO.

SÁNCHEZ-CRIADO, T. y LÓPEZ, D. (2007): "La Necesidad de Adaptar las TIC para la Promoción de la Autonomía en el Marco de la Ley de Dependencia". Revista de Formación y Empleo, n 91, pp. 5-10.

SANCHO CASTILLEJO, M. y DÍAZ MARTÍN, R. (2007): "Atención a las Personas Mayores en su Entorno: Teleasistencia, Ayuda a Domicilio y Centros de Día". Sociedad y Utopía: Revista de Ciencias Sociales, n 30, pp. 161-190.

SINGER, M. y DONOSO, P. (2007): "Assessing an ambulante service with queuing theory". Computers \& operations research, n 35 , pp. 1-22.

SINGER, M., DONOSO, P. y SCHELLER-WOLF, A. (2008): "Una introducción a la teoría de colas aplicada a la gestión de servicios". Revista ABANTE, $\mathrm{n}^{\circ}$ 11 (2), pp. 1-22.

TAHA, H. A. (1997): Investigación de Operaciones, una Introducción. México: Prentice Hall.

TIRADO, F., LÓPEZ, D., CALLÉN, B. y DOMÉNECH, M. (2008): "La Producción de Fiabilidad en Entornos Altamente Tecnificados. Apuntes Etnográficos sobre un servicio de Teleasistencia Domiciliaria". Papeles del CEIC, $\mathrm{n}^{\circ} 2$ (38), pp. 1-28. 
